COLABORACIÓN ESPECIAL

\title{
PROGRAMA Y COBERTURAS DE VACUNACIÓN FRENTE A SARAMPIÓN Y RUBEOLA EN ESPAÑA. RETOS PARA ALCANZAR SU ELIMINACIÓN
}

\section{Aurora Limia Sánchez y Marta Molina Olivas.}

Subdirección General de Promoción de la Salud y Epidemiología. Dirección General de Salud Pública, Calidad e Innovación. Ministerio de Sanidad, Servicios Sociales e Igualdad.

Las autoras declaran no tener conflictos de interés.

\section{RESUMEN}

Una de las estrategias fundamentales establecidas por la Organización Mundial de la Salud para conseguir la eliminación del sarampión y la rubeola es alcanzar y mantener coberturas de vacunación muy elevadas con dos dosis frente a sarampión y al menos una dosis frente a rubeola en el programa de vacunación rutinario. El programa actual de vacunación en España contempla la administración de dos dosis de vacuna combinada frente a sarampión, rubeola y parotiditis a los 12 meses y los 3-4 años de edad. Desde el año 2000 se mantienen las coberturas iguales o superiores al $95 \%$ con la primera dosis, pero con la segunda dosis las coberturas son menores, entre el $90-95 \%$. En el año 2014 no se ha alcanzado el objetivo para la primera dosis en tres comunidades autónomas y para la segunda dosis solamente ocho comunidades autónomas han alcanzado el objetivo. Se discuten los retos y aspectos a mejorar para fortalecer el programa de vacunación con la finalidad de conseguir la eliminación de estas enfermedades en España. .

Palabras clave: Brotes epidémicos. Sistema de información. Sarampión. Rubéola. Vacunación masiva. Vacuna contra el sarampión.

Correspondencia

Aurora Limia Sánchez

alimia@msssi.es

DOI:

\section{ABSTRACT \\ Immunization Programme and Coverage against Measles and Rubella in Spain. \\ Challenges for Achieving their Elimination}

The World Health Organization had established the achievement and sustainability of very high coverage with two doses of vaccine against measles and at least one against rubella as one of the key strategies for the elimination of both measles and rubella. The current immunization programme in Spain includes the immunization with two doses of combined vaccine against measles, mumps and rubella at 12 months and 3-4 years of age. Since 2000 coverage with first dose is over the target of $95 \%$ but the coverage with the second dose remains between 90 and $95 \%$. In 2014, at subnational level three regions had coverage below the objective and only eight regions achieved the objective for the second dose. The challenges and some activities to strengthen the immunization programme in order to achieve the elimination of measles and rubella are discussed.

Keywords: Outbreaks. Information system. Measles. Rubella.Vaccines. Vaccination. Mass vaccination. Measles vaccine. 


\section{INTRODUCCIÓN}

En el año 2010, el Grupo Asesor de Vacunación de la Organización Mundial de la Salud (OMS) realizó una amplia revisión sobre la posibilidad de erradicación del sarampión y concluyó que esta enfermedad puede y debe erradicarse, y recomendó la factibilidad de eliminación conjunta del sarampión y la rubeola ${ }^{1}$. Utilizando la experiencia de la Región de las Américas, que eliminó ambas enfermedades, la Región Europea estableció las estrategias fundamentales para su consecución. Alcanzar y mantener altas coberturas de vacunación con dos dosis en el programa rutinario establecido en los diferentes niveles territoriales es uno de los pilares fundamentales para la eliminación del sarampión y la rubeola ${ }^{1}$.

En este artículo se realiza una introducción de la política de vacunación en España y el establecimiento del calendario de vacunación y, de manera más detallada, la descripción de la evolución del programa de vacunación frente a sarampión y a rubeola en España. Se presenta la evolución de las coberturas con vacuna frente a sarampión, rubeola y parotiditis (triple vírica), las coberturas en el año 2014 y se discuten los retos del programa para conseguir la eliminación del sarampión y la rubeola en España.

\section{POLÍTICA DE VACUNACIÓN}

Desde el traspaso de las competencias sanitarias del Estado a las comunidades autónomas (CCAA) a partir del año 1981, las administraciones sanitarias competentes en la gestión del programa de vacunación son las CCAA, del establecimiento de sus respectivos calendarios de vacunación y de la compra, distribución y administración de las vacunas.

El Consejo Interterritorial del Sistema Nacional de Salud (CISNS) es el órgano permanente de coordinación, cooperación y comunicación entre la Administración del Estado y las $\mathrm{CCAA}^{2}$. Su misión es coordinar todos los programas de salud, tanto asistenciales como de salud pública, entre los que se encuentran los programas de vacunación. Del CISNS dependen diversas Comisiones, Comités y Grupos de Trabajo y Ponencias ${ }^{3}$. Una es la Comisión de Salud Pública de la que depende la Ponencia del Programa y Registro de Vacunaciones, organismo técnico asesor del CISNS en materia de vacunas y política de vacunación, que tiene representación territorial. Esta Ponencia propone recomendaciones para España teniendo en cuenta el conocimiento científico y la epidemiología de las enfermedades prevenibles mediante vacunación. Las decisiones del CISNS se adoptan por consenso y, por lo tanto, no son vinculantes, aunque se espera que todas las CCAA incorporen las decisiones adoptadas puesto que participan en su discusión, siempre y cuando no haya razones epidemiológicas que justifiquen la diferencia.

\section{CALENDARIO DE VACUNACIÓN}

El calendario oficial de vacunación es el aprobado por el CISNS y por las autoridades sanitarias de las CCAA. Es de aplicación universal y voluntaria y abarca desde el nacimiento hasta los 14 años de edad. Los costes de las vacunas y su administración son cubiertos por el Sistema Nacional de Salud. Las modificaciones en el calendario de vacunaciones se realizan en base al procedimiento acordado en el seno del CISNS $^{4}$ tras lo cual se incorporan en todas las CCAA. Aunque adicionalmente algunas deciden de manera unilateral la administración de otras vacunas, las diferencias actuales son mínimas 5 .

Aunque con anterioridad el calendario de vacunación se actualizaba cuando se realizaba alguna modificación en él, desde el año 2012 se actualiza anualmente incorporando los cambios que se hayan acordado en el seno del CISNS. El calendario común de vacunación infantil para el año 2015, acordado por el CISNS el 14 de enero de 2015, incluye el programa de vacunación sistemática con vacuna frente a sarampión, rubeola y parotiditis (vacuna triple vírica) con la primera dosis ad- 
ministrada a los 12 meses y la segunda a los 3 o 4 años de edad (figura 1).

\section{PROGRAMA DE VACUNACIÓN FRENTE A SARAMPIÓN Y RUBEOLA}

La vacunación frente a sarampión se introdujo en España en el año 1968, tras el éxito alcanzado con las campañas de vacunación frente a poliomielitis y difteria, tétanos y tos ferina, que comenzaron a partir del año 1964. La campaña de vacunación frente a sarampión se inició en once provincias, vacunando a todos los niños entre los 9 y los 24 meses de edad. La vacuna utilizada contenía como principio activo la cepa Beckenham 31. Esta vacuna resultó ser muy reactogénica, ocasionando efectos adversos en el $10 \%$ de los niños vacunados, por lo que se dejó de vacunar. Se retiró del registro nacional en 1970, auto- rizándose en 1975 la vacuna que contenía la cepa hiperatenuada de Schwarz?

El mismo año 1975 se instauró el primer calendario de vacunación en sustitución de las campañas de vacunación, incluyéndose en 1977 la vacuna frente a sarampión a los 9 meses de edad y la monovalente frente a rubeola en las niñas a los 11 años de edad. Ambas vacunas comenzaron a administrarse en España en 1978.

En 1981 se sustituyó en el calendario la vacuna frente a sarampión por la vacuna triple vírica, (sarampión, rubeola y parotiditis), que comenzó a administrarse a los 15 meses y se continuó vacunando a las niñas a los 11 años de edad frente a rubeola ${ }^{8}$.

La administración de la segunda dosis con vacuna triple vírica comenzó a administrarse

\section{Figura 1}

Calendario de vacunación acordado para el año 2015. Consejo Interterritorial del Sistema Nacional de Salud, enero 2015

CONSE JO INTE RTERRITORIAL DEL SISTEMA NACIONAL DE SALUD

CALENDARIO COMÚN DE VACUNACIÓN INFANTIL

Calen dario recomendado an 02015

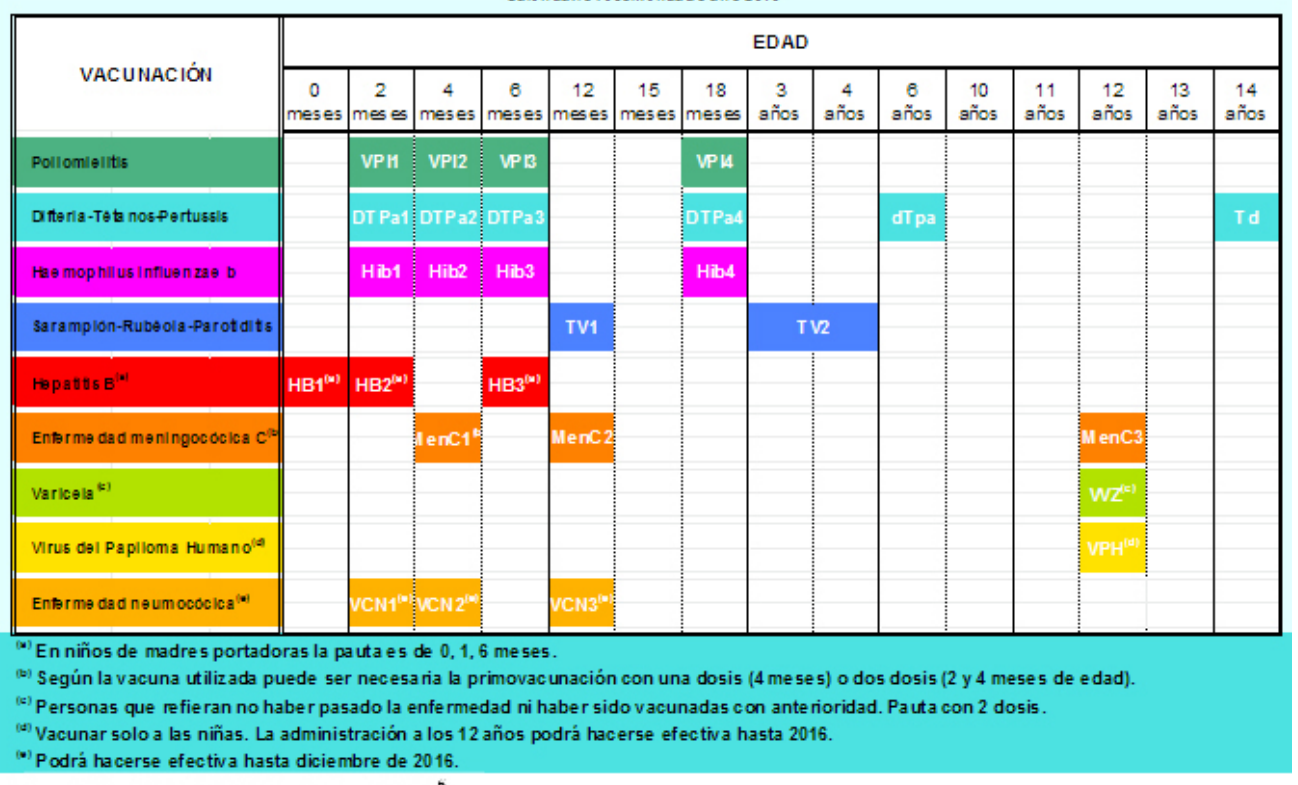

Fuente: Ministerio de Sanidad, Servicios Sociales e Igualdad ${ }^{b}$ 


\begin{tabular}{|c|c|c|}
\hline \multicolumn{3}{|c|}{$\begin{array}{c}\text { Tabla } 1 \\
\text { Vacunas frente a sarampión y rubeola actualmente disponibles en España }\end{array}$} \\
\hline & Priorix (GSK) & M-M-RVAXPRO (SPMSD) \\
\hline Presentación & $\begin{array}{l}\text { Polvo y disolvente } \\
\text { Jeringa precargada }\end{array}$ & $\begin{array}{l}\text { Polvo y disolvente } \\
\text { Jeringa precargada }\end{array}$ \\
\hline Composición & $\begin{array}{l}\text { S: cepa Schwarz } \\
\text { R: cepa Wistar RA 27/3 } \\
\text { P: RIT4385 (derivada Jeryl-Lynn) } \\
\text { Trazas de neomicina } \\
\text { Contiene sorbitol }\end{array}$ & $\begin{array}{l}\text { S: cepa Ender' Edmonston } \\
\text { R: cepa Wistar RA 27/3 } \\
\text { P: cepa Jeryl-Lynn } \\
\text { Trazas de neomicina } \\
\text { Contiene sorbitol }\end{array}$ \\
\hline Administración & Vía subcutánea (intramuscular) & Vía subcutánea o intramuscular \\
\hline
\end{tabular}

a todos los niños y niñas de 11 años de edad en sustitución de la vacunación frente a rubeola que se administraba solamente a las niñas. El momento de incorporación de este cambio fue diferente en cada CCAA, consecuencia del traspaso de competencias en Salud Pública entre los años 1979 y 1985, siendo Cataluña la primera en introducirla en 1988. En 1994 se había implementado esta segunda dosis de triple vírica a los 11 años en 12 CCAA.

En 1996 se acordó el primer calendario de vacunación del CISNS, el cual incluía la primera dosis de vacuna frente a sarampión, rubeola y parotiditis entre los 12 y 15 meses y la segunda dosis entre los 11 y 13 años de edad, incorporándose en los programas de vacunación de todas las CCAA.

En 1999 se adelantó la segunda dosis a los 3-6 años de edad tras el análisis del estudio seroepidemiológico realizado en España en 1996, que mostró baja seroprotección en los niños de 6 a 9 años de edad ${ }^{8}$.

En 2012 se realizó el último cambio en la edad de administración de ambas dosis tras la observación de que los brotes de sarampión durante el periodo 2010-2012 incluían un importante número de casos en niños entre los 12 y los 15 meses de edad y la mayor parte de las CCAA establecían la edad de vacunación a los 15 meses. Se adelantó la primera dosis a los 12 meses y la segunda a los 3-4 años de edad, y así permanecen en el calendario vigente para el año 2015 (figura 1).

Actualmente, en España no se dispone de vacunas monovalentes frente a sarampión ni rubeola. Se dispone de dos vacunas combinadas para la vacunación frente a sarampión, rubeola y parotiditis (tabla 1).

\section{COBERTURAS DE VACUNACIÓN FRENTE A SARAMPIÓN Y RUBEOLA}

Las coberturas de vacunación reflejan la información recogida de forma rutinaria sobre las vacunas administradas. Las CCAA recogen y analizan la información sobre las vacunas administradas a nivel local mediante registros numéricos o nominales. Anualmente, en base al Acuerdo del CISNS de 22 de Junio de $1992^{10}$, notifican al Ministerio de Sanidad la población diana para la administración de cada vacunación y las personas vacunadas en su territorio. Tras su análisis en el Ministerio y validación por las CCAA, se publican en la página web del Departamento ${ }^{11}$. Adicionalmente, desde él se envía información sobre coberturas de vacunación solicitadas anualmente por la OMS y otros organismos internacionales y nacionales, tanto de forma regular como de manera específica para estudios y objetivos concretos, como son el seguimiento del proceso de erradicación de la poliomielitis y la eliminación del sarampión y la rubeola. 
Figura 2

Evolución de las coberturas de vacunación frente a sarampión y rubeola. España, 2000-2014

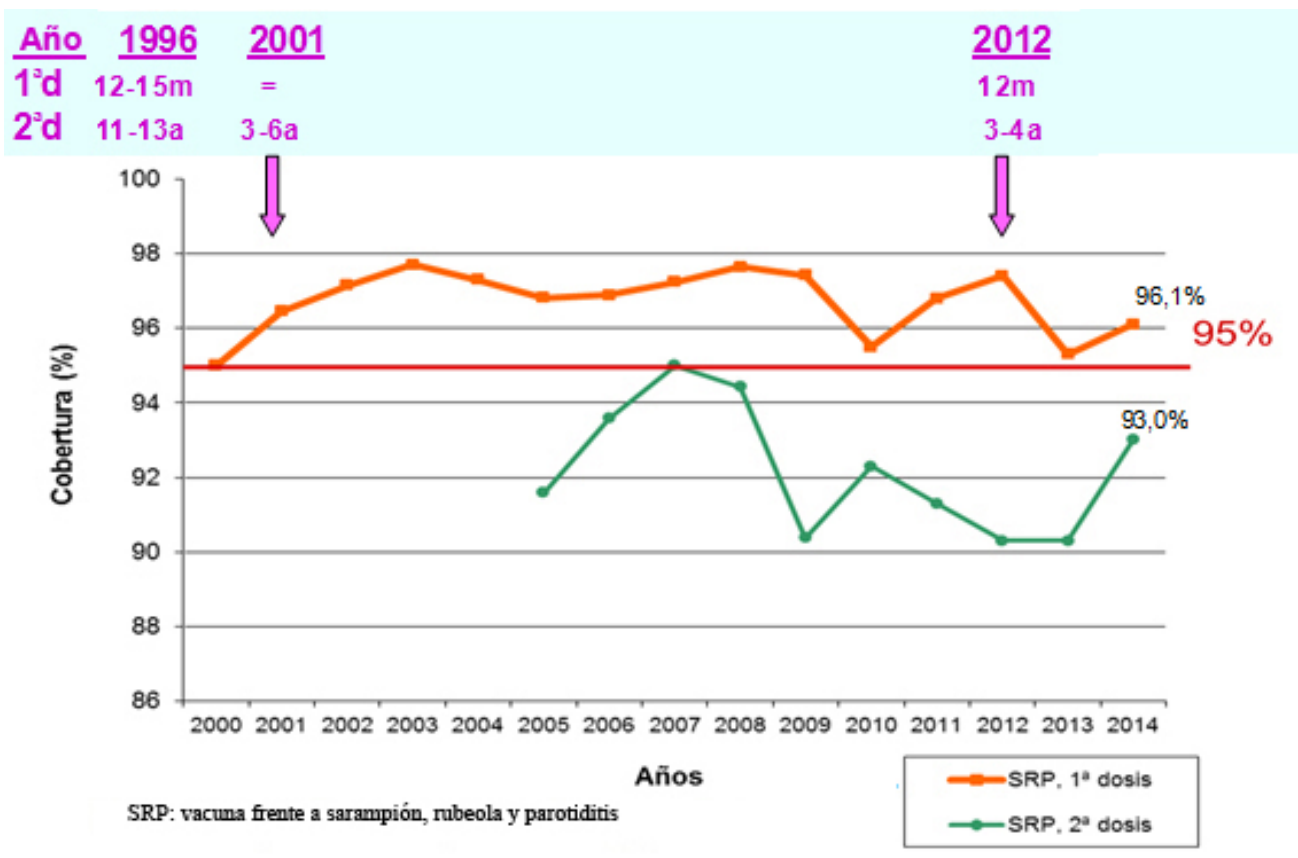

El objetivo del programa de vacunación frente a sarampión y rubeola es alcanzar una cobertura del 95\% con dos dosis, en consonancia con el establecido desde la OMS para la eliminación de ambas enfermedades ${ }^{1}$.

Desde el comienzo de la vacunación sistemática con vacuna triple vírica en 1978, la cobertura de vacunación ha ido aumentando $^{12,13}$, siendo superiores al 95\% desde el año 2000. A nivel de España se dispone de información de coberturas de vacunación con la segunda dosis de vacuna triple vírica desde el año 2005. La cobertura con esta segunda dosis se ha mantenido desde entonces superior al 90\%, alcanzándose el objetivo del 95\% solamente en 2007 (figura 2).

La cobertura de vacunación en 2014 fue del $95,3 \%$ con la primera dosis y del $90,7 \%$ con la segunda, con un intervalo de coberturas entre las CCAA de 90,9-100\% y de $83,1-100 \%$ para la primera y segunda dosis, respectivamente. Por lo tanto, se observa que, a nivel de España, se alcanza el objetivo de vacunación con la primera dosis, pero la cobertura de la segunda está por debajo del objetivo del 95\%. Sin embargo, el objetivo para alcanzar la eliminación del sarampión y la rubeola es la cobertura de vacunación igual o superior al 95\% con dos dosis en la población diana de vacunación rutinaria ${ }^{1}$.

Las coberturas de vacunación en las CCAA en el año 2014 se muestran en la figura 3. Ceuta y Melilla y 14 CCAA tuvieron coberturas de vacunación con la primera dosis superiores al 95\% y 3 CCAA entre el 90 y el $95 \%$. La cobertura de vacunación con la segunda dosis es igual o superior al $95 \%$ en 8 $(42,1 \%)$ CCAA, 7 (36,8\%) tienen coberturas entre $90-94 \%$ y $4(21,1 \%)$ entre $80-89 \%$.

En los últimos años algunas CCAA están realizando cambios en los registros de vacunación que pueden repercutir en la obtención 


\section{Figura 3}

\section{Coberturas de vacunación en comunidades autónomas. Año 2014}

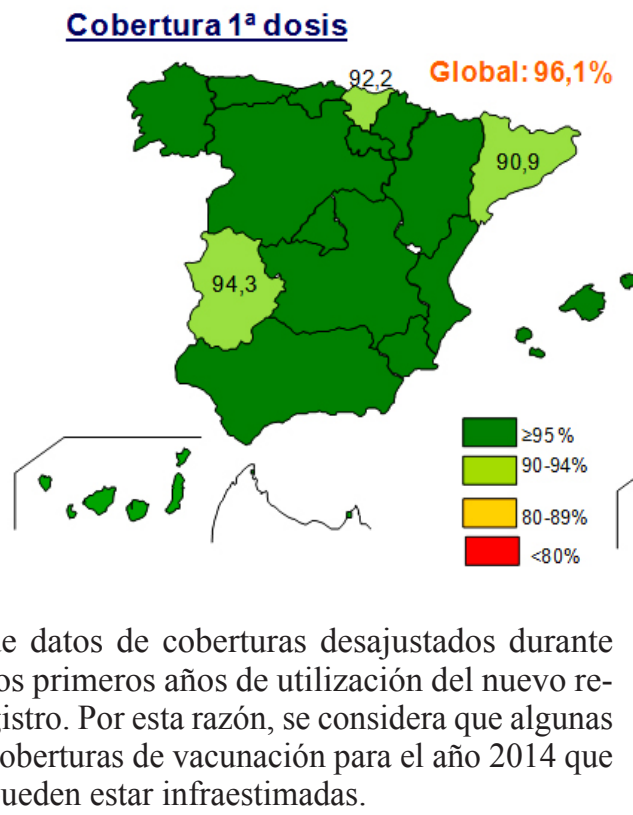

DISCUSIÓN Y RETOS PARA LA ELIMINACIÓN DEL SARAMPIÓN Y LA RUBEOLA

La vacuna atenuada frente a sarampión es muy efectiva, produciéndose seroconversión en el $95 \%$ o más de las personas vacunadas a partir de los 12 meses de edad. Casi todos los niños que no desarrollan inmunidad tras la administración de la primera dosis lo hacen tras administrarles la segunda, asegurándose tasas de seroconversión del $95 \%$ o mayor si la primera dosis se administra a los 9 meses y del $99 \%$ o más si la primera dosis se administra a partir de los 12 meses. Como resultado de la alta transmisibilidad del virus del sarampión, el umbral para alcanzar inmunidad comunitaria es muy elevado, requiriéndose coberturas de vacunación igual o superiores al 95\% para interrumpir su transmisión ${ }^{14-17}$.

Más del $95 \%$ de las personas vacunadas con una sola dosis de virus atenuados frente a rubeola tiene protección frente a viremia y frente a la enfermedad durante al menos 15 años $y$, probablemente durante toda la vida ${ }^{1,17}$. Además, el virus de la rubeola es menos transmisible que el del sarampión, por lo que el umbral para alcanzar inmunidad comunitaria es menor del $90 \%{ }^{15,18}$.

Las coberturas de vacunación reflejan la protección de la población diana con la vacunación sistemática. Aunque las actualmente alcanzadas con dos dosis de vacuna podrían ser aceptables para el control del sarampión, no lo son para alcanzar la eliminación de la enfermedad. El número de personas susceptibles que van quedando cada año como resultado de no vacunarse y de no inmunizarse tras vacunación con una sola dosis van acumulándose, formando una bolsa de sujetos susceptibles creciente que puede favorecer la transmisión en el momento de la entrada de un virus importado.

En el caso de rubeola, las coberturas de vacunación alcanzadas en España con una dosis de triple vírica son apropiadas para alcanzar la eliminación de esta enfermedad desde los primeros años de la vacunación rutinaria en la infancia. 
El calendario de vacunación sistemática está bien establecido en España y, en general, a juzgar por las coberturas de vacunación en la infancia, está bien aceptado por parte de los profesionales sanitarios y la población. El Ministerio de Sanidad y las CCAA deben trabajar conjuntamente en el fortalecimiento coordinado del programa de vacunación frente a sarampión y rubeola adaptándolo a los requerimientos establecidos por la OMS para conseguir la eliminación de ambas enfermedades.

Es necesario mejorar las coberturas de vacunación con ambas dosis de triple vírica, pero sobre todo con la segunda. Se debe asegurar que la información sobre coberturas de vacunación es homogénea y fiable. El desarrollo de sistemas de información de coberturas de vacunación ha sido heterogéneo en las CCAA, siendo deseable disponer de sistemas de información que permitan monitorizar la cobertura de vacunación en tiempo real. El conocimiento de la protección de la población frente a sarampión y rubeola mediante estudios de seroprevalencia, como fuente de información adicional sobre el perfil inmunitario de la población, puede ayudar en la toma de decisiones, por lo que es deseable realizar un nuevo estudio para conocer la evolución desde la anteriormente realizada en el año 1996.

Exceptuando la actuación durante brotes epidémicos, en general, en España no se realizan actividades complementarias de vacunación. Se considera necesario tener conocimiento de las características de la población susceptible y de las razones por las que hay personas que no se vacunan, así como desarrollar de manera coordinada estrategias de vacunación dirigidas a grupos de vacunación específicos ${ }^{1}$. La finalidad última es proteger a toda la población y prevenir la transmisión de la enfermedad en caso de importación del virus.

Un aspecto importante es la sensibilización de la población y de los profesionales sanitarios sobre la importancia de la factibilidad de la eliminación del sarampión y la rubeola y sobre los beneficios y los riesgos de la vacunación y de las enfermedades.

Es de gran importancia la sensibilización de los responsables políticos sobre el objetivo de eliminación y la necesidad de contar con los recursos apropiados para establecer las actividades necesarias para conseguir la eliminación del sarampión y la rubeola en España.

Como conclusión, aunque se considera que el nivel de aceptación del programa de vacunación frente a sarampión y rubeola en la edad infantil es bueno, se considera necesario fortalecer el programa en línea con los criterios establecidos por la OMS para conseguir la eliminación de estas enfermedades en España.

\section{AGRADECIMIENTOS}

A los miembros de la Ponencia de Programa y Registro de Vacunaciones y a los gestores del programa de vacunación en las comunidades autónomas.

\section{BIBLIOGRAFÍA}

1. Eliminating measles and rubella. Framework for the verificacion process in the WHO European Region. World Health Organization. Regional Office for Europe, 2014. [citado el 23 de julio de 2015].

Disponible en: http:/www.euro.who.int/_data/assets/ pdf_file/0009/247356/Eliminating-measles-and-rubellaFramework-for-the-verification-process-in-the-WHOEuropean-Region.pdf?ua=1 2. Boletín Oficial del Estado. Ley 14/1986, de 25 de abril, General de Sanidad. BOE núm. 102, de 29-04-1986.

3. El Consejo Interterritorial del Sistema Nacional de Salud. Ministerio de Sanidad, Política Social e Igualdad. [citado el 16 de julio de 2015] Disponible en: http:// www.msps.es/organizacion/consejoInterterri/home.htm.

4. Grupo de Trabajo Criterios 2011, de la Ponencia de Programa y Registro de Vacunaciones. Criterios de evaluación para fundamentar modificaciones en el Programa de Vacunación en España. Comisión de Salud Pública del Consejo Interterritorial del Sistema Nacional de Salud. Ministerio de Sanidad, Política Social e Igualdad. 2011. [citado el 23 de julio de 2015] Disponible en: http://www.msssi.gob.es/profesionales/saludPublica/ prevPromocion/vacunaciones/docs/Criterios_ProgramaVacunas.pdf. 
5. Ministerio de Sanidad, Servicios Sociales e Igualdad. Calendarios de vacunación recomendados en España. Consejo Interterritorial y comunidades y ciudades autónomas. [citado el 23 de julio de 2015] Disponible en: http:// www.msssi.gob.es/ciudadanos/proteccionSalud/infancia/ vacunaciones/programa/vacunaciones.htm

6. Consejo Interterritorial del Sistema Nacional de Salud. Calendario común de vacunación infantil recomendado para el año 2015. [citado el 23 de julio de 2015] Disponible en: http://www.msssi.gob.es/ciudadanos/proteccionSalud/ vacunaciones/docs/CalendarioVacunacion2015.pdf

7. Pachón del Amo I. Historia del programa de vacunación en España. En: Amela C. Epidemiología de las enfermedades incluidas en un programa de vacunación. Monografía de la Sociedad Española de Epidemiología. 2004. [citado el 23 de julio de 2015] Disponible en: http://www. seepidemiologia.es/documents/dummy/monografia1_vacunas.pdf.

8. Amela Heras C, Pachón del Amo I. Estudio seroepidemiológico: situación de las enfermedades vacunables en España. Instituto de Salud Carlos III, 2000. [citado el 23 de julio de 2015] Disponible en: http://www.isciii.es/ ISCIII/es/contenidos/fd-servicios-cientifico-tecnicos/fdvigilancias-alertas/fd-enfermedades/SEROEPIDEMIOLOGICO.pdf

9. Vacuna triple vírica (sarampión, rubeola, parotiditis). Vacunas de uso humano autorizadas en España, combinadas víricas. Agencia Española de Medicamentos. [citado el 23 de julio de 2015] Disponible en: http://www.aemps. gob.es/medicamentosUsoHumano/vacunas/autorizadasEspana/comb_Viricas.htm

10. Consejo Interterritorial del Sistema Nacional de Salud. Acuerdo número 192. Pleno 22 junio 1992. Disponible en: http://www.msssi.gob.es/organizacion/consejoInterterri/docs/192.pdf [citado el 23 de julio de 2015].

11. Ministerio de Sanidad, Servicios Sociales e Igualdad. Coberturas de vacunación. [citado el 23 de julio de 2015] Disponible en: http://www.msssi.gob.es/profesionales/saludPublica/prevPromocion/vacunaciones/coberturas.htm

12. Plan de eliminación del sarampión en España. Instituto de Salud Carlos III, febrero de 2000. [citado el 23 de julio de 2015] Disponible en: http://www.isciii.es/ISCIII/es/ contenidos/fd-servicios-cientifico-tecnicos/fd-vigilanciasalertas/fd-enfermedades/fd-enfermedades-preveniblesvacunacion/PLANSARAMPION.pdf

13. de la Torre Misiego JL. Cobertura vacunal en España. Rev Esp Salud Pública.1999; 73: 617-618.

14. Amela C. Programas de vacunación: modificaciones en la dinámica de la transmisión. En: En: Amela C. Epidemiología de las enfermedades incluidas en un programa de vacunación, capítulo 5. Monografía de la Sociedad Española de Epidemiología. 2004. [citado el 23 de julio de 2015] Disponible en: http://www.seepidemiologia.es/ documents/dummy/monografia1_vacunas.pdf
15. Strebel PM, Papania MJ, Dayan GH, Halsey NA. Measles Vaccine. En Plotkin S, Orenstein W, Offit P, editors. Vaccines. Fifth Edition. Atlanta: Saunders Elsevier; 2008. p. 353-398.

16. Package for accelerated action: 2013-2015. World Health Organization. Regional Office for Europe, 2014. [citado el 23 de julio de 2015] Disponible en: http://www. euro.who.int/_data/assets/pdf_file/0020/215480/PACKAGE-FOR-ACCELERATED-ACTION-20132015.pdf

17. Fine P, Eames K, Heyman DL. "Herd immunity": a rough guide. Clinical Infectious Diseases, 2011; 52(7): 911-916.

18. Plotkin SA, Reef SE. Rubella Vaccine. En Plotkin S, Orenstein W, Offit P, editors. Vaccines. Fifth Edition. Atlanta: Saunders Elsevier;2008. p. 735-771. 